\title{
«HAGO DE TODO Y NO SÉ HACER FUNCIONAR NADA»: APRENDIZAJE AFECTIVO Y RELACIONAL DE TECNOLOGÍAS DIGITALES EN ADULTOS MAYORES
}

\section{"I do everything, but I don't know how to make anything work»: Affective and relational learning of social media in later life}

Roser Beneito-Montagut

Cardiff University. Reino Unido

Correo-e: beneito-montagutr@cardiff.ac.uk

Arantza Begueria

Universität Siegen. Alemania

Correo-e: arantzazu.begueria@gmail.com

Nizaiá Cassián

Universitat Oberta de Catalunya

Recepción: ro de diciembre de 2017

Envío a informantes: 14 de diciembre de 2017

Aceptación definitiva: 20 de enero de 2018

Resumen: Este artículo exploratorio argumenta que tratar el aprendizaje de los adultos mayores únicamente como adquisición de «conocimiento representacional» no es adecuado si queremos entender cómo aprenden a usar las tecnologías digitales en general y las tecnologías de medios de comunicación social en particular. Usando ejemplos tomados de un estudio etnográfico que explora el uso de las redes sociales de los adultos mayores, se presentan una variedad de prácticas digitales y relaciones afectivas a través de las cuales ocurre el aprendizaje. La idea de comunidades de práctica es un enfoque útil, sin embargo, también debe ampliarse para tener en cuenta las prácticas que ocurrieron fuera de los centros sociales y culturales y fuera de los grupos de interés formados en torno al aprendizaje de tecnologías digitales. También es útil responder a la pregunta de

* Esta investigación ha sido financiada por Acup/Recercaixa. 
cómo y por qué las formas particulares de «autoridad pedagógica» se promulgan a través de prácticas digitales particulares, que son altamente relacionales y afectivas, y cómo estas formas de experiencia se convierten en rasgos de identidad. Luego discutimos las implicaciones de esto para nuestra comprensión de la identidad y el envejecimiento dentro de la sociedad digital. Finalmente, sugerimos que el término ecologías de aprendizaje capta mucho mejor las prácticas de aprendizaje de los adultos mayores.

Palabras clave: aprendizaje; medios de comunicación social; adultos mayores; afectos; relaciones; tecnologías digitales.

АвSTRACт: This exploratory paper argues that treating older adults learning as «representational knowledge» acquisition is not adequate if we want to understand how they learn to use digital technologies in general, and social media technologies in particular. Using examples taken from an ethnographic study exploring social media use in later life, the case is made for a range of digital practices and affective relations through which learning happens. The idea of communities of practice is a useful approach, however it also needs to be expanded to account for the practices that happened outside of the social and cultural centers and outside of the groups of interest formed around the interests in digital technologies. It is also useful to answer the question of how and why particular forms of "pedagogical authority» are enacted through particular digital practices, which are highly relational and affective, and how these forms of expertise become identity traits. We further discuss the implications of this for our understandings of identity and ageing within the digital society. Finally, we suggest that the term learning ecologies captures much better the practices of learning of older adults.

KEY WORDs: learning; social media; later life; affects; relationships; digital technologies.

\section{Introducción}

$\mathrm{D}$ ESDE LA POPULARIZACIÓN DE INTERNET y las tecnologías de la información y comunicación el acceso de los mayores a la sociedad digital era limitado y crecía poco a poco. Sin embargo, recientemente ha habido un aumento del acceso a esta, fomentado por la popularidad de los dispositivos móviles (teléfonos y tablets) y su amplia adopción por los mayores adultos (Diduca et al., 2007; Fernández-Ardèvol y Prieto, 20I2; Madden, 20IO; Tsai et al., 20I5). En este contexto, resulta pertinente volver a preguntarse cómo aprenden tecnologías digitales los mayores adultos en su día a día. La búsqueda de una respuesta a esta pregunta empieza por la introducción del planteamiento conceptual y teórico de esta investigación.

Hay un reconocimiento general de la idea de que el uso de Internet y las tecnologías de la información y la comunicación (TIC) es esencial para la creación de sociedades contemporáneas más justas e igualitarias (por ejemplo, Policies for Ageing Well with ICT, 20I0; Timmers, 2008). Hasta la fecha, los estudios que examinan el uso y aprendizaje de estas tecnologías por parte de adultos mayores se han realizado desde perspectivas de adopción, abordando aspectos de motivación, barreras y percepciones de utilidad (por ejemplo, Selwyn, 2004; Selwyn et al., 2003), o, por otro lado, de accesibilidad y aprendizaje (Jaeger y Xie, 2009; Leist, 20I3), pero poniendo el foco en los problemas o dificultades que este grupo social tiene para interactuar completa 
«HAGO DE TODO Y NO SÉ HACER FUNCIONAR NADA»: APRENDIZAJE AFECTIVO

Y RELACIONAL DE TECNOLOGÍAS DIGITALES EN ADULTOS MAYORES ROSER BENEITO-MONTAGUT, ARANTZA BEGUERIA Y NIZAIÁ CASSIÁN

y significativamente con las tecnologías. Desafortunadamente, esta tendencia es generalizada. Tal y como describe Boulton-Lewis: «una gran cantidad de investigación sobre el aprendizaje entre adultos mayores está, lamentablemente, dominada por la identificación de obstáculos y la disminución en el proceso (de aprendizaje) causado por un deterioro físico y mental» (2010: 217).

Con todo, pocos estudios han analizado, con detalle y en profundidad, cómo los adultos mayores usan y aprenden nuevas tecnologías en contextos no formales, cotidianos y relacionales. Previos artículos se han limitado a estudiar cómo aprenden los mayores a partir de experiencias de aprendizaje concretas, y centrados en la adquisición de competencias según parámetros establecidos por otros grupos sociales, no por ellos. Este tipo de aprendizaje es lo que Sørensen llama «conocimiento representacional» (2009) y se refiere al aprendizaje basado en la adquisición de conocimiento que ya está ahí, en los libros de texto o en las políticas sociales, y que es generalmente instrumental -tiene un fin determinado, por ejemplo, adquirir ciertas competencias o habilidades que son útiles en puestos de trabajo-. Además, este tipo de aprendizaje se suele estudiar o adquirir fuera de contextos cotidianos, en escuelas, universidades o centros de formación. Sin embargo, el aprendizaje tácito e invisible que está inscrito en actividades mundanas raramente emerge en estos estudios.

Del mismo modo, generalmente, se ha tendido a utilizar marcos teóricos desde la pedagogía con una aproximación claramente cognitiva. Distanciándose de estas perspectivas, recientemente se ha realizado un conjunto de estudios sobre aprendizaje que busca alejarse de la preocupación por los individuos, los maestros o las mentes, para dedicarse al estudio de la situación (situatedness), el contexto de aprendizaje y sus interrelaciones (como Aberton, 2012; Fenwick y Landri, 2012; Mathisen y Nerland, 2012; Sørensen, 2009). Estos estudios investigan el aprendizaje situado, relacional y afectivo (Wetherell, 20I2), a la vez que proponen un descentramiento del enfoque educativo en el sujeto humano individual, para centrarse en el proceso de aprendizaje. Evaden, por tanto, la supremacía de las concepciones representacionalistas del conocimiento e inician una exploración de las diversas formas en que el aprendizaje y el conocimiento se arraigan en las prácticas, incluyendo la acción continua que producen los objetos y las subjetividades que constituyen nuestra forma de ver el mundo. Estas formas de conocimiento incluyen el estudio de la acción continua que producen los objetos y materialidades presentes en las situaciones de aprendizaje. Al mismo tiempo, intentan ir más allá de nociones limitadas de "participación» y "comunidad de práctica» (Wenger, 1998a), entendidas como una estructura social para un fin determinado. La característica distintiva es el enfoque en la participación como elemento central para el aprendizaje. De hecho, en la teoría de Lave y Wenger participar es aprender (2002). El concepto de participación puede ser utilizado para investigar tanto el compromiso con la práctica social y de aprendizaje, como la forma en que estas prácticas de aprendizaje se conectan para desarrollar un historial de aprendizaje que cambia la relación con la práctica, con otros actores sociales involucrados en la misma, y como parte de esto, se relaciona con su subjetividad/identidad. Sin embargo, como hemos indicado, esta noción de «comunidad de practica» es limitada y ha sido ampliamente criticada (Hughes et al., 2013). Las críticas se refieren en parte al dominio de ciertos actores sociales en la construcción social de las propias prácticas -de aprendizaje y de uso de las redes de comunicación social- y, finalmente, en la producción de conocimiento. También es crítico, desde nuestra perspectiva, el foco en los actores 
«HAGO DE TODO Y NO SÉ HACER FUNCIONAR NADA»: APRENDIZAJE AFECTIVO

Y RELACIONAL DE TECNOLOGÍAS DIGITALES EN ADULTOS MAYORES ROSER BENEITO-MONTAGUT, ARANTZA BEGUERIA Y NIZAIÁ CASSIÁN

humanos, desatendiendo el papel «no neutro» de la tecnología. El posicionamiento teórico de este artículo parte de estas posiciones críticas. En nuestro caso, podemos afirmar que los jóvenes -o también aquellos problemáticamente llamados «nativos digitales»- dominan las prácticas en red y promulgan la construcción social de las mismas.

También, en este marco teórico, es necesario introducir la idea de prácticas como las unidades de análisis de la investigación y, más concretamente, la idea de aprendizaje alrededor de las prácticas digitales de los adultos mayores. Para nosotras, la noción de prácticas hace referencia no únicamente a lo que ocurre en los medios sociales digitales, se refiere también a aquello que sucede fuera del ámbito digital, pero en relación con este. La idea de prácticas puede conceptualizarse grosso modo como las redes que incluyen actores humanos (tales como iguales, familiares, amigos, profesores), sus actividades, usos e interacciones sociales y cualquier tipo de recursos (como herramientas, artefactos, modelos, normas sociales, objetivos) que los actores humanos utilizan. Consecuentemente, estamos interesadas en estudiar las dimensiones de la vida social que son emergentes -el uso de medios de comunicación social o redes sociales- y que están menos articuladas en teorías previas y que, a su vez, su estudio nos permite el desarrollo de narraciones más dinámicas, atractivas y enérgicas (Wetherell, 20I2).

Dentro de este marco conceptual, aprender es entendido como una práctica y un proceso. Siguiendo la definición de Sørensen (2009), quien reivindica que el aprendizaje es un crecimiento en conocimiento y la habilidad lograda de conectarse con otras entidades, aprender «a ser» también es un proceso continuo que ocurre a través de cruces y ensamblajes de materiales-discursivos e interacciones de personas, lugares, cuerpos, textos, tecnologías, artefactos y arquitecturas. En resumen, este enfoque desafía la suposición convencional de que el aprendizaje es principalmente un logro individual, como hemos indicado anteriormente.

En este artículo mostramos, a partir de viñetas, varias prácticas de aprendizaje diversas y relacionales. Para ello, nos basamos en un estudio etnográfico de las prácticas cotidianas de un grupo de adultos mayores en centros sociales en Barcelona y en un pueblo de ámbito rural en la provincia de Barcelona. Con ello pretendemos hacer visibles las particulares microdinámicas cotidianas del aprendizaje. Además, ponemos el foco en la relacionalidad como elemento altamente imbricado en los procesos de aprendizaje en torno a las prácticas digitales en redes de comunicación social. También ponemos el acento en el objeto tecnológico -como los teléfonos móviles y los diferentes productos que se activan con el uso de la tecnología digital- y en las acciones/relaciones sobre dichos objetos que promulgan los discursos sobre la edad y la vejez en relación a las tecnologías. En otras palabras, el artículo describe un amplio rango de encuentros pedagógicos y situaciones sociales alrededor del aprendizaje tecnológico y de las redes de comunicación social. El objetivo final del artículo es reexaminar conceptos de aprendizaje y sus vínculos con la formación de identidades en torno a dos ejes: edad y tecnología.

\section{Metodología}

Este artículo está basado en los datos generados para un proyecto de investigación etnográfico que examina el uso de los medios sociales en la vida cotidiana de los adultos 
«HAGO DE TODO Y NO SÉ HACER FUNCIONAR NADA»: APRENDIZAJE AFECTIVO

Y RELACIONAL DE TECNOLOGÍAS DIGITALES EN ADULTOS MAYORES

ROSER BENEITO-MONTAGUT, ARANTZA BEGUERIA Y NIZAIÁ CASSIÁN

mayores. En términos de la orientación metodológica para este proyecto, se realizó una etnografía digital en equipo (Beneito-Montagut et al., 20I7), siguiendo el enfoque clásico de Erickson y Stull (1998), que considera la etnografía en equipo un modelo válido gracias a su configuración como proceso deliberativo.

La investigación planteó una serie de preguntas con el objetivo de explorar cómo las prácticas de la vida cotidiana de los adultos mayores en las redes sociales ayudan a construir y mantener relaciones sociales. Asimismo, también pretendía investigar las relaciones cotidianas, «enredadas» e imbricadas entre el uso de las redes sociales y el aislamiento social.

El objetivo final consistió en describir las cualidades y materialidades de las relaciones sociales y las redes sociales. Aunque el foco inicial del proyecto no era el aprendizaje, al iniciarse el trabajo de campo en centros sociales y en los cursos sobre redes de comunicación social, fue notable la aparición de diversos resultados en torno a prácticas de aprendizaje de tecnologías en general, y de medios sociales en red en particular.

El proyecto siguió una metodología multisituada y centrada en el usuario pero utilizando las prácticas como unidades de análisis, para llevar a cabo un trabajo de campo basado en observación participante tanto en línea como fuera de línea, que también incorporaba entrevistas en profundidad y el análisis de datos digitales multimedia (Beneito-Montagut, 20II).

La etnografía tuvo lugar en Cataluña, en cuatro centros comunitarios para adultos mayores y a través de varias aplicaciones de redes sociales, así como en los hogares de los participantes y espacios públicos como cafeterías. Usando los centros comunitarios como un punto de acceso, 20 usuarios de medios sociales de más de 64 años (II hombres y 9 mujeres) participaron en el estudio. La interacción online con estos participantes comenzó justo después de una primera entrevista. En dichas primeras entrevistas, se recabó información sobre las prácticas cotidianas en los medios sociales, los cursos sobre informática a los que habían asistido y las relaciones personales de los participantes, en un sentido amplio. Interactuar con los participantes en línea implicó estar presentes en todos los servicios y plataformas de redes sociales que utilizaban (principalmente Facebook, WhatsApp y correo electrónico, aunque una pequeña cantidad de los participantes también usaban Twitter e Instagram) durante los dos años que duró el trabajo de campo. También se recolectaron datos digitales de las redes sociales sobre las prácticas digitales en línea de los participantes. Durante un período de cinco semanas, se recopiló la actividad de las redes sociales de cada usuario, tanto en forma de base de datos, que incluye metadatos, como en forma de datos visuales de capturas de pantalla convertidas en PDF. La etnografía terminó con una última entrevista con cada participante. Todos los datos generados y recolectados fueron incluidos en un programa informático de análisis de datos cualitativos para su posterior codificación y análisis por parte de las investigadoras.

Las viñetas que se presentan a continuación se centran en describir las prácticas de aprendizaje tecnológico en diferentes situaciones y representan diferentes historias en torno a las relaciones sociales y a la edad. En términos pragmáticos, entendemos una viñeta como una descripción de una serie de eventos escogidos por ser representativos, típicos o emblemáticos de las prácticas de aprendizaje tecnológico de los adultos mayores que participaron en la investigación. Cada viñeta tiene una estructura narrativa similar a una historia que se limita a un breve lapso de tiempo, a uno o unos pocos 
actores clave y a un espacio delimitado (Miles y Huberman, 1994). Su pertinencia, tanto epistemológica como metodológica, radica en una comprensión de las mismas como historias vívidas de las prácticas (Erickson, 1985).

\section{Aprendiendo tecnología y uso de redes de comunicación social en comunidades de prácticas}

\section{I. Eva y el aprendizaje en los centros socioculturales}

Eva: No, ya sabes por qué te lo digo también, ¿no? Yo me bajo muchas del Youtube, $¿$ eh? Porque me las bajo en MP3, y entonces me las paso a WAW a vav, porque para el PowerPoint tiene que ser música VAV, porque normalmente si es MP3 si se lo envías según como, no se oye la música, y por vav siempre se oye la música, y entonces la transformo.

E: Es que sois, sois ... unos especialistas, ¿eh? Realmente...

Eva: ¿Por qué?

E: De, de todo este tema del audio y del vídeo, y...

Eva: A ver, es que hacemos cursos. Yo hago cursos. Yo de hecho doy clases de PowerPoint, y doy clase de vídeo, y de Photoshop.

Eva es una mujer de 72 años que vive en Barcelona, está retirada -era administrativa- y vive con su marido. Eva se pasa gran parte del día en el centro sociocultural de su barrio, en el que además es coordinadora del área de informática. Está muy implicada y vinculada a la comunidad en torno al centro sociocultural. Tiene una vida social muy activa, y gran parte de su actividad está vinculada a este centro que se encuentra en su barrio, donde participa en muchísimos de los cursos que se ofrecen, no solo en aquellos relacionados con las tecnologías. Como ella misma dice, "ya sabes que yo estoy muy ocupada, que estoy aquí en el centro sociocultural, y tengo mucho trabajo». Cuando llega a casa por la noche, después de cenar, suele conectarse a Internet durante tres horas, hasta que se va a la cama.

Su interés por las tecnologías digitales también está articulado alrededor del centro sociocultural. Tiene dos teléfonos móviles, uno que no es smartphone y un smartphone que comparte con su marido por razones económicas. En casa tienen un ordenador de sobremesa, un portátil y una tablet. Usa correo electrónico y Facebook. Aunque su perfil de Facebook está más bien «abandonado» y no hay demasiada actividad. Sin embargo, Eva se encarga de actualizar la página de Facebook de la asociación, y en este espacio sí que es bastante activa y dinámica. Ahí publica todas las noticias del centro sociocultural, actividades, fotos e información interesante para la gente que acude y participa en el centro. Actúa como una especie de «comunity manager» para el centro sociocultural.

Ha probado un alto número de aplicaciones diferentes, como WhatsApp, Twitter e incluso creó su propio blog, pero posteriormente lo abandonó y dejó de utilizar la mayoría de las aplicaciones. Es de las personas que prueba todo lo que cae en sus manos, y después abandona aquello que no acaba integrándose en su rutina diaria. Sus prácticas habituales se articulan en torno al correo electrónico y al Facebook de la comunidad del centro, que no ha abandonado y, según ella, pasa 3 horas al día conectada. Eva utiliza la página del centro como si se tratase de su Facebook personal. Sin embargo, reconoce que lo que más utiliza es el correo electrónico. 
«HAGO DE TODO Y NO SÉ HACER FUNCIONAR NADA»: APRENDIZAJE AFECTIVO Y RELACIONAL DE TECNOLOGÍAS DIGITALES EN ADULTOS MAYORES ROSER BENEITO-MONTAGUT, ARANTZA BEGUERIA Y NIZAIÁ CASSIÁN

Como muchas de las personas que han participado en el estudio, Eva está muy interesada en los cursos sobre redes sociales -e informática en general- que se ofrecen en los centros socioculturales. Los cursos de formación que le interesan están muy relacionados con el uso de los móviles y con las prácticas digitales visuales y multimedia, como vemos en la cita que abre la sección. Por medio de los cursos en los centros sociales, Eva y sus compañeros aprenden cómo hacer vídeos, PowerPoints interactivos y multimedia. Observamos en Eva, y en varios de los participantes de la investigación, que existe una fascinación por las prácticas visuales y multimedia que impulsa la voluntad de aprendizaje de herramientas para la edición digital multimedia. Para otros, destapa una admiración por aquellos que son capaces de realizarlos. Esta admiración les otorga una especie de reconocimiento como expertos. Sin embargo, estas prácticas no terminan ahí, porque el objetivo final de las mismas es compartir los vídeos y los PowerPoints, normalmente a través de cadenas de mails, pero también en Facebook o YouTube. Poder compartirlos es muy importante para ellos, como observamos en el fragmento de conversación con el que abrimos la sección. De nuevo, en palabras de Eva, «hicimos un PowerPoint, y bueno, cuando lo terminamos nos lo enviamos [entre el grupo que asiste al curso]». Este hecho, además de reforzar esta idea de construcción de la pericia por medio de la práctica, nos apunta también hacia la relevancia de los elementos relacionales de las mismas. Y a cómo los productos tecnológicos que han realizado se convierten en objetos de intercambio simbólico que forman parte de la construcción de la identidad de los participantes en relación al resto de los miembros del grupo.

De algún modo, lanzarse a aprender cómo hacer PowerPoints animados -o vídeos-, y su laboriosa creación, tiene también como finalidad el poder compartirlos y mostrar lo que se ha sido capaz de realizar -a pesar de que dicho reconocimiento no se da fuera del grupo de adultos mayores-. El artefacto tecnológico, y las competencias que se demuestran con él, se convierten en un aspecto que añade una dimensión identitaria al creador. En este artículo, entendemos que las identidades no pueden ser nunca definitivamente inmovilizadas (Segal, 2008). Deben ser vistas como fundamentalmente contingentes y únicamente estabilizadas temporalmente a través de actos e interacciones sociales en determinados contextos socioculturales. Identidad, de este modo, es una categoría de la que subyace la idea de subjetividad. Cuando argumentamos que la realización de los productos tecnológicos se convierte en un elemento identitario, nos referimos a su función como marcadores identificadores, pero nuestro objetivo es subrayar la multiplicidad de formas de mostrarlos que opera dentro de un imaginario sociocultural determinado y que promulga un proceso de identificación/desidentificación a través de la tecnología, alejado de posiciones binarias estables. En las siguientes secciones seguiremos desarrollando cómo las ideas de pericia y subjetividades, están asociadas a los modos en los que la vida material y digital es organizada en el contexto de las prácticas de aprendizaje y cómo afectan a la construcción social de la edad.

\subsection{Pere realizando y compartiendo un PowerPoint}

Pere, de 70 años, tiene dos ordenadores, uno de ellos portátil, y es también una persona especialmente fascinada por las prácticas multimedia. Normalmente pasa 
conectado a Internet dos o tres horas al día y es muy hábil en Facebook y en tecnologías multimedia. Asiste asiduamente a cursos de informática en centros cívicos y sociales. Ahora se ha convertido en una de las personas más admiradas en su centro habitual por sus conocimientos de tecnologías multimedia, que comparte con los demás un día a la semana, en que la sala de ordenadores queda abierta para todos los usuarios y Pere actúa de voluntario para ayudar a los demás en sus dudas. Una mañana en el centro de mayores, entre consulta y consulta, se dedica a hacer un PowerPoint con fotografías de jugadores del FC Barcelona. Para ello, descarga fotografías de distintas
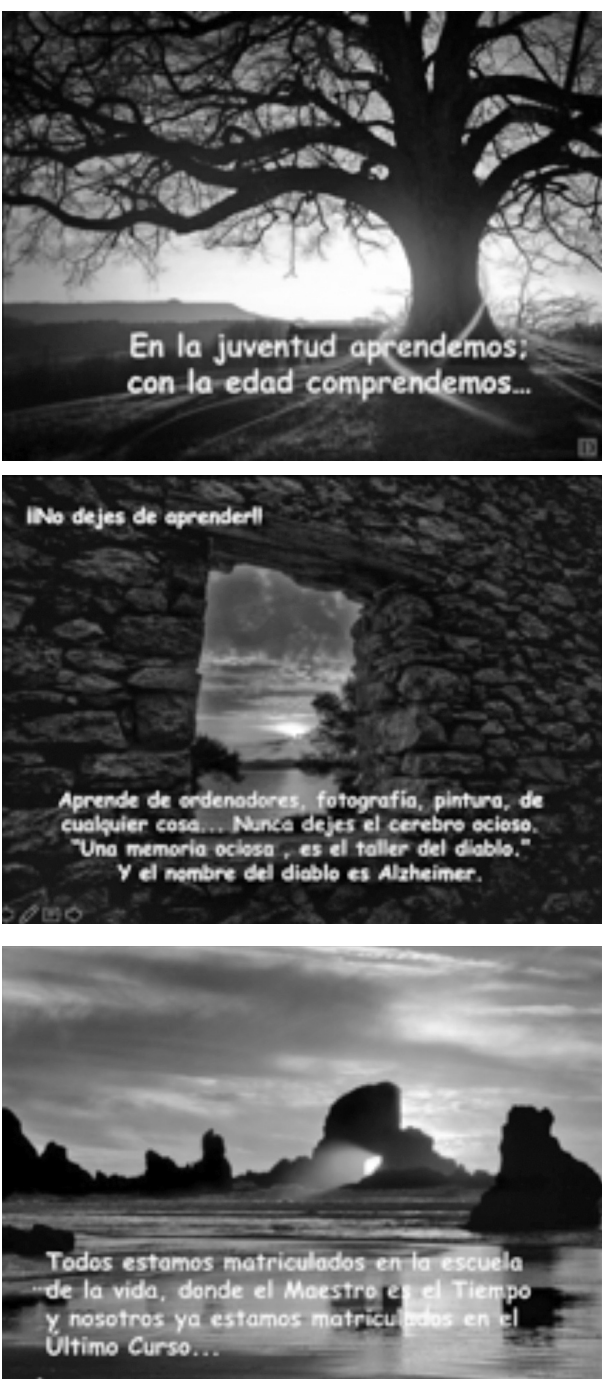

Figura i: Secuencias de un PowerPoint creado por uno de los participantes reflexionando en torno a la edad. páginas web, las modifica con Photoshop para que cambien de color e incluye animaciones. Finalmente, busca incansablemente páginas web para descargar el himno del Barça, que también quiere incluir en la presentación. Para ello, tendrá que hacer una conversión de formato de la canción, que realiza con un programa que también ha descargado de Internet. La elaboración de la presentación es lenta y laboriosa y la realiza ante la atenta mirada y el entusiasmo de los demás usuarios de la sala, que también hacen sugerencias para mejorarla. Una vez acabada la presentación, muy orgulloso de ella, la manda a sus amigos por mail y por el chat privado de Facebook, volviendo de nuevo a remarcar la relevancia de los aspectos relacionales. Una práctica ligada a la realización de PowerPoints, y que pone de relieve los aspectos identitarios que acarrean estos objetos en cuanto visualizadores de la pericia, es la protección de los mismos con contraseñas para que ningún otro usuario pueda apropiarse de estas creaciones, adaptarlas, modificarlas y hacerlas pasar por propias.

La Figura I muestra un ejemplo de los PowerPoints que realizan. Precisamente este reflexiona sobre el envejecimiento y el aprendizaje. Su visión sobre la edad es algo que aparece frecuentemente en las conversaciones, en las que aprender se percibe como algo muy positivo y como una práctica que sucede a lo largo de la vida.

También sirve para ilustrar lo que se considera conocimiento válido y habilidades relevantes entre los adultos mayores que participan de estas prácticas de 
«HAGO DE TODO Y NO SÉ HACER FUNCIONAR NADA»: APRENDIZAJE AFECTIVO Y RELACIONAL DE TECNOLOGÍAS DIGITALES EN ADULTOS MAYORES ROSER BENEITO-MONTAGUT, ARANTZA BEGUERIA Y NIZAIÁ CASSIÁN

aprendizaje. Los PowerPoints son expresiones que visibilizan importantes aprendizajes cotidianos: estas visualizaciones plantean preguntas críticas sobre cómo se configura esta autoridad pedagógica y cómo otorga valor a determinados conocimientos mientras que ignora otros (Fenwick y Landri, 20I2).

\subsection{Eva, los centros socioculturales y la red como espacios de socialización}

Los cursos y talleres organizados para aprender y adquirir competencias digitales ejercen también de espacios de socialización, como ya hemos observado en las prácticas descritas hasta el momento. Podemos extender esta idea sobre los centros como espacios de socialización también al ámbito de la Red. Esta socialización se extiende también a las redes de comunicación social, y esto abre posibilidades adicionales de relacionalidad. Como explica Eva, «... además aquí hacemos cursillos de ... de las redes sociales, "La red del día a día”, pues entonces la gente que se apunta, todos se dan de alta en el Facebook. Y luego al principio recibo un montón de solicitudes de amistad y yo claro, como son gente de aquí los acepto». Esto es algo que se observa constantemente durante el trabajo de campo en los centros culturales, «bueno, es que aquí todos estamos conectados, la mayoría estamos conectados a Internet eh ... cosas de ... de informática con las cosas aquí. Aquí viene, venimos y la mayoría de gente pasamos muchas horas aquí dentro».

Sin embargo, cuando prestamos atención a la relación entre el uso de redes sociales de comunicación y el aumento de las relaciones interpersonales no encontramos una relación clara entre su uso y el aumento. En todo caso, quizá se produce una intensificación de relaciones personales y un contacto más frecuente con la gente que ya se conoce y con la que ya se ha establecido algún tipo de vínculo fuera de las redes sociales. En este sentido, las tecnologías son un elemento más (digital y material) de y para la socialización, que refuerza la generación de comunidades de aprendizaje, pero por ellas mismas no aumentan las relaciones sociales. Eva aprende y enseña simultáneamente a través de su compromiso con el centro sociocultural y los cursos sobre tecnologías. Sus actividades en el centro social y en las redes online llevan a Eva a tener nuevos contactos, nuevas relaciones y nuevas prácticas, pero no es el hecho de que sean nuevos contactos lo que los hace relevantes, sino los aspectos relacionales y subjetivos asociados con las prácticas. Para Eva, los PowerPoints que se mandan en cadena o los vídeos que sube a Youtube también actúan como una extensión o demostración de sus habilidades y pericias digitales. Del mismo modo, la página de Facebook y los correos electrónicos que regularmente responde para ayudar en temas tecnológicos a otros miembros de la comunidad -por ejemplo, sobre cómo incorporar audio a un PowerPoint- son una confirmación de su estatus como "experta» y líder dentro de la comunidad de aprendizaje.

\subsection{Teresa y su aprendizaje como miembro de un grupo de iguales}

El siguiente escenario que presentamos ilustra las prácticas de aprendizaje que se producen entre iguales. Teresa tiene 69 años, es viuda y vive también en Barcelona. Tiene 3 hijos y 4 nietos, pero vive sola. Ella tiene y utiliza todo tipo de aparatos 
«HAGO DE TODO Y NO SÉ HACER FUNCIONAR NADA»: APRENDIZAJE AFECTIVO Y RELACIONAL DE TECNOLOGÍAS DIGITALES EN ADULTOS MAYORES ROSER BENEITO-MONTAGUT, ARANTZA BEGUERIA Y NIZAIÁ CASSIÁN

tecnológicos: ordenador de sobremesa, portátil, tablet y smartphone. Cuando hablamos con ella sobre sus prácticas digitales cotidianas nos cuenta que tras enviudar pasó un tiempo bastante difícil intentando situarse, paralizada por el sentimiento de pérdida, pero en los cursos de informática del centro sociocultural cercano a su casa encontró algo que le interesaba mucho y que le apetecía hacer. De ese modo, Teresa empezó hace 6 años a participar en los cursos de formación relacionados con la tecnología de su centro social. Ya desde el principio, las prácticas digitales de Teresa tuvieron un fuerte elemento relacional, afectivo y emocional.

Y entonces, a raíz de ahí, en aquella época, la página de La Caixa tenía un chat. Un chat. Pero sólo de escribir, porque entonces aún no, no usábamos el Skype y todos estos que nos veíamos. Y por este chat de La Caixa, hice dos o tres amigos, y que después nos... bueno, por el chat. Pero gente jubilada, también. Un viudo aquí, uno de allí, no sé qué. Que después, detrás de este... «oye, ponte el Skype, que así nos veremos, que nos conoceremos, que no se-qué». Y nos pusimos el Skype y el Messenger, que también tenía videoconferencia. Y pues mira... este me pasaba programas.

Fue el interés de Teresa por las tecnologías informáticas y por aprender a usarlas lo que funcionó como catalizador o motivación para establecer relaciones sociales. Ella empezó a ir al centro porque le interesaba la tecnología, y eso funcionó como centro de actividad para formar un grupo, y de ahí, surgieron relaciones sociales relevantes para ella. Esto sucedió hace varios años. Ahora, este grupo de adultos mayores es conocido entre los centros socioculturales de Barcelona por ser el grupo de «expertos» en temas informáticos. Por este motivo, al centro habitual de Teresa acuden varias personas que no son del barrio y que se desplazan para asistir a las clases con ellos. Por tanto, la motivación inicial de las personas que actualmente acuden a las clases fue a la vez tecnológica y relacional, porque querían conocer y estar en contacto con otras personas con intereses comunes.

Teresa aprendió, y sigue aprendiendo, como parte de un grupo de iguales que comparten intereses, y se relacionan tanto en el centro social como a través de las redes sociales, y esto también le ha dado la confianza y la experiencia para ser ella la que enseña a su amiga Paca.

Un día observamos cómo Teresa, que maneja muy bien las redes y diversos programas informáticos, ayuda a una amiga. Teresa decide traer su ordenador portátil y ponerse a publicar algo en Facebook con su amiga. Paca, la amiga, se bloquea y dice «que a mí no me sale» y "que no lo sabré hacer». Tiene miedo porque no sabe qué hacer e insiste «me parece que no lo sabré hacer», «¡dame los apuntes!». Teresa, después de esta escena con la amiga, nos cuenta que Paca «se bloquea mucho, se pone nerviosa, de tantos apuntes que hace después no se aclara con lo que apunta. Bueno, yo a veces estoy con ella al teléfono una hora, con el ordenador abierto: "va, ve aquí, clica allá”». Sin embargo, a pesar de estas tensiones, a Paca también le gusta mucho Internet y disfruta de las relaciones sociales a través de Internet. Aprovecha para seguir hablando de un amigo común de la dos que vive en Canarias que es un cracker y les pasa programas informáticos pirateados. De nuevo Teresa nos explica: «Y... claro, siempre me explicaba los problemas que él tenía [refiriéndose a problemas personales de salud], yo, “¿Y cómo haces [refiriéndose a un proceso tecnológico]? ¿Cómo has encontrado esto?”, ¿sabes? Teníamos... eso». Teresa, con estas historias sobre sus relaciones, ilustra de manera ejemplar cómo las cuestiones 
«HAGO DE TODO Y NO SÉ HACER FUNCIONAR NADA»: APRENDIZAJE AFECTIVO

Y RELACIONAL DE TECNOLOGÍAS DIGITALES EN ADULTOS MAYORES

ROSER BENEITO-MONTAGUT, ARANTZA BEGUERIA Y NIZAIÁ CASSIÁN

tecnológicas se enredan con las relaciones sociales y se genera afectividad e intimidad alrededor de estas prácticas de aprendizaje. Durante el trabajo etnográfico encontramos ejemplos recurrentes de este tipo de prácticas que hemos llamado de aprendizaje entre iguales, que la mayoría de las veces tienen un carácter emocional y suponen un sentimiento de cercanía.

La idea de una comunidad de prácticas (Lave y Wenger, I99I; Wenger, I998b) sintetiza lo que hemos encontrado al observar cómo se construye el aprendizaje situado en los centros sociales. Una comunidad de práctica es un grupo que participa en una experiencia compartida. Los inexpertos en esa práctica son considerados «expertos en desarrollo» a través de la participación en actividades legítimas y reconocidas -cursos de formación, «making» de objetos digitales (desde PowerPoints a vídeos), ser un «cracker» o simplemente aportar a una actividad en red (como publicar un post o una imagen en Facebook)- que contribuyen a la práctica, pero que no son necesariamente centrales, como lo sería un elemento evaluable en otros procesos de aprendizaje formales. Gradualmente, estas contribuciones se vuelven más complejas e importantes por medio de la participación. Sin embargo, es necesario ampliar la noción de comunidad de prácticas para dar cuenta de las relaciones afectivas, que consideramos centrales en el proceso de aprendizaje, y de las relaciones materiales y digitales que se establecen con los objetos y artefactos tecnológicos.

\section{De aprender a enseñar}

A continuación, veremos una escena que describe un tipo de contribuciones más complejas que emergen dentro y a partir de las comunidades de prácticas y que ilustran cómo un grupo de adultos mayores pasan de ser aprendices a ser maestros. La diferencia entre esta viñeta y la anterior radica en que el identificador subjetivo de pericia se reafirma al situarse en otro contexto fuera de los centros socioculturales de los diferentes barrios.

En uno de los centros socioculturales, algunos participantes, que se han convertido en reconocidos expertos para el grupo, se han movido gradualmente del rol de aprendices al de maestros. Como hemos visto, los aprendices progresivamente ensamblan la idea general de lo que constituye la práctica en sí. Eva ha pasado de ser una aprendiz de tecnologías digitales a ser ella misma quien enseña a otros, al igual que Teresa. Sin embargo, encontramos un grupo de participantes cuyas prácticas van más allá e incluso enseñan a otros de modo más organizado. Este grupo, que surge en un centro sociocultural y a partir del interés por los temas informáticos, imparte y participa de lo que ellos mismos han llamado cursos de autoformación. Un día a la semana, son ellos mismos los que imparten las clases en función de la pericia de cada persona. Es decir, aquel o aquella que es bueno/a editando imágenes con Photoshop imparte una sesión sobre eso. Y, así, sucesivamente con diversas prácticas, ya que la mayoría de las sesiones se organizan alrededor de cómo hacer algo específico.

José tiene 80 años, y vive con su mujer. Tiene un ordenador de sobremesa, una tablet y un smartphone. Es muy activo en Internet y en diversas redes sociales. Es muy hábil en el manejo de herramientas de edición digital (como Photoshop, PowerPoint y edición de vídeo) y es voluntario para dar cursos sobre tecnología e informática tanto en el centro sociocultural al que asiste habitualmente, como en un centro de 
«HAGO DE TODO Y NO SÉ HACER FUNCIONAR NADA»: APRENDIZAJE AFECTIVO Y RELACIONAL DE TECNOLOGÍAS DIGITALES EN ADULTOS MAYORES ROSER BENEITO-MONTAGUT, ARANTZA BEGUERIA Y NIZAIÁ CASSIÁN

integración social para mujeres sin hogar y en una cárcel de Barcelona. Además, también ayuda en una asociación de consumidores. Como él mismo nos explica:

El tema de Internet lo he ido aprendiendo en cursillos, y preguntando a compañeros. Nos lo vamos explicando, nos autoformamos, con compañeros que también son voluntarios de la Fundación de La Caixa. Y nos autoformamos un día a la semana. Y entonces, pues es... Los conocimientos que tengo son los que transmito a otras personas que vienen a los cursillos. Yo les doy clases de informática [refiriéndose a las mujeres del centro de integración social]. Les enseño. Todas son principiantes o sea que no saben ni abrir un correo ni nada. Les he enseñado a abrir un correo, algo de Power Point, cositas así. Tomar fotos de Internet, ponerlas, etc.

Durante el trabajo de campo en el centro sociocultural pudimos observar cómo José actúa y practica un liderazgo tecnológico. Se sabe y es reconocido como experto, lo cual le confiere una autoridad pedagógica. Ayuda constantemente a todo el mundo. José es realmente un líder -desde la perspectiva de autoridad pedagógica-. Es el que más sabe e incluso sus movimientos corporales en el aula de informática atienden a este papel dentro del grupo. Controla los horarios de ocupación de las aulas y de las clases, organiza el uso de las mismas, hace callar a la gente -con tono autoritariocuando se hacen corrillos durante las clases, dispone los turnos de palabra y todo el mundo le pregunta dudas. Además, es el que se encarga de mandar los recordatorios y las fichas de cada sesión. Es líder, tanto en Internet como fuera de Internet, dado que todos esperan sus instrucciones.

Esta serie de viñetas nos ayudan a comprender varios aspectos emergentes en las prácticas de los adultos mayores en torno al aprendizaje y el uso de tecnologías digitales en el día a día. En primer lugar, hemos observado cómo las subjetividades en torno a las prácticas de aprendizaje son construidas, más concretamente hemos visto cómo aspectos identitarios relacionados con la idea de pericia están arraigados al uso y la habilidad a la hora de manejar las tecnologías. Segundo, hemos visto cómo se construyen y sostienen las comunidades de prácticas y, finalmente, cómo se entienden las prácticas mismas y los mecanismos por los cuales se las piensa como válidas. Lo interesante respecto a las comunidades de prácticas de los adultos mayores, es que, en primer lugar, no nos sirven nociones tradicionales relacionadas con una finalidad instruccional de las mismas, y, por ello, es necesario superar nociones tradicionales de comunidades de prácticas. En segundo lugar, cabe remarcar la importancia de los aspectos relacionales y afectivos que están implícitos en las mismas. Finalmente, también cabe remarcar el hecho de que dichas comunidades se generan principalmente en espacios físicos (materiales), pero se expanden e intensifican con las redes de comunicación social.

\section{Otros modos de aprendizaje}

En la sección anterior hemos analizado cómo se entienden y construyen las prácticas de aprendizaje digitales, cómo surgen y cómo funcionan dentro de las comunidades de prácticas, siendo nuestro principal argumento que surgen y se organizan a partir de las prácticas mismas, que son relacionales y están cargadas de afectividad. Además, estas prácticas y los niveles de pericia o habilidad forman parte de procesos 
«HAGO DE TODO Y NO SÉ HACER FUNCIONAR NADA»: APRENDIZAJE AFECTIVO Y RELACIONAL DE TECNOLOGÍAS DIGITALES EN ADULTOS MAYORES ROSER BENEITO-MONTAGUT, ARANTZA BEGUERIA Y NIZAIÁ CASSIÁN

subjetivos y funcionan como marcadores identitarios al margen de la edad. La tecnología no es neutral en estos procesos de construcción social de las prácticas de aprendizaje. Sin embargo, antes de avanzar a analizar la construcción de edad en relación con el aprendizaje de tecnologías digitales, queremos mencionar -aunque no analizaremos con detalle- dos modos de aprendizaje que nos parecen significativos (y recurrentes durante trabajo de campo).

El primero hace referencia al relevante papel del autoaprendizaje. Bernat, de 66 años y que vive en Barcelona con su pareja, tiene smartphone, un Netbook y portátil. Nos explica que un elemento importante en el aprendizaje de las tecnologías ha sido «muchas horas, y muchas horas y muchas horas». Él pasa de 2 a 3 horas cada día haciendo cosas con el ordenador. Esta experiencia se repite en los participantes del proyecto, que se descargan manuales de uso de programas, han aprendido a usar vídeos tutoriales de Youtube que les guían en la realización de tareas, siguen tutoriales sobre cómo editar vídeo, etc. Y, además, comparten todos estos recursos. El segundo modo de aprendizaje que queremos brevemente mencionar es el aprendizaje intergeneracional, basado principalmente en las relaciones entre padres/madres e hijos/as y abuelos/as y nietos/as. Son continuas las referencias que los participantes hacen a las prácticas de aprendizaje intergeneracional y son también reiteradas las referencias a las tensiones que surgen en estas relaciones -principalmente en las relaciones con los hijos-. Bernat nos cuenta:

- Bernat: Pero este señor, este señor es mayor, este señor tiene, este señor debe de tener ahora 73 años aproximadamente. Y si, él sí que está metido en todo: WhatsApp, tiene, nosequé, pam. ¡Y claro, tiene las nietas estas que lo hacen bailar (ríe), lo hacen, pero bueno! (ríe). Y entonces, pues sí, sí, cuando tiene algún problema, llama a la niña [refiriéndose a la nieta]. El otro día mismo, estábamos allí en la oficina de la cooperativa esta, que es bah, algo que hacemos, y queríamos poner una foto con el WhatsApp y ¡no sabíamos cómo hacerlo! ¡Ni él, ni el otro señor, ni yo, nadie!

-E: Sí, he visto que tú no tenías.

- Bernat: Y cogió aquella, dice -Llama a la nieta y dice «escucha, cómo se pone la fotografía aquí?». (Baggg) le dijo "pero qué teléfono?». «No sé, un teléfono que tiene aquí, no se qué». «Bueno, pues mira esto, mira aquello». Y entonces, cogí yo, digo «a ver, mujer las instrucciones: pum, pim, pim» ¡y al final pudimos hacerlo!!! ¡Pudimos hacerlo!! Y claro, colgamos, dijo «ime cago en la niña de I2 años, que nos dé todo esto, que tal!!

El argumento principal que intentamos traer aquí es que, aunque hemos descrito y argumentado que emergen comunidades de prácticas relacionadas con el aprendizaje de tecnologías, estas no son la única forma de aprendizaje, ya que aprender pasa por hacer y practicar regularmente (horas y horas, en palabras de Bernat), y por preguntar a gente «más joven». Los jóvenes son percibidos por los participantes como «expertos» en tecnología y redes sociales por el mero hecho de ser jóvenes. Por ello, los participantes recurren frecuentemente a ellos, preguntan cómo hacer determinadas cosas en las redes de comunicación social. Curiosamente, no hemos encontrado ningún caso en el que algún participante pregunte a hijos/as o nietos/as algo relacionado con cómo realizar PowerPoints. En cambio, sí que hemos observado, aunque no de un modo recurrente, el elemento relacional y afectivo que hemos descrito anteriormente, y que se desarrolla principalmente entre iguales, en relación a las comunidades de prácticas. 
«HAGO DE TODO Y NO SÉ HACER FUNCIONAR NADA»: APRENDIZAJE AFECTIVO

Y RELACIONAL DE TECNOLOGÍAS DIGITALES EN ADULTOS MAYORES ROSER BENEITO-MONTAGUT, ARANTZA BEGUERIA Y NIZAIÁ CASSIÁN

\section{Aprendizaje y discursos «edadistas»}

Conscientemente, hemos esquivado hasta ahora los discursos sobre edad que surgen en las prácticas. La razón de huir de esto es porque el análisis se ha centrado en las prácticas, más que en los discursos, y en ellas no son obvias las visiones «edadistas», más allá del PowerPoint que hemos utilizado como ejemplo y que mostraba una visión extremadamente positiva de la relación edad/aprendizaje. El «edadismo», a diferencia de otros tipos de discriminación, es un prejuicio y un estigma (Goffman, 2006) que pasa generalmente inadvertido, quizá porque es un modo de «ser»y «estar» al que casi todos llegaremos, independientemente de otro tipo de discriminaciones por género, raza, clase social, discapacidad, etcétera.

Sin embargo, eso no significa que los discursos y perspectivas edadistas no existan y que no hayan aparecido durante el trabajo etnográfico. De hecho, podemos observar alguno de estos prejuicios en el fragmento de conversación con Bernat, que hemos citado arriba. De las palabras de Bernat se desprende que la edad es una cuestión relacional, se es joven o viejo respecto a otro, no es una cuestión fija, estática e inmutable. Bernat tiene 66 años y caracteriza como mayor a alguien de 73, cuando habla sobre la pericia en el manejo de las redes de comunicación social y los smartphones. Pero lo que queremos remarcar es la figuración implícita en sus palabras de que a mayor edad mayor dificultad para manejar las tecnologías digitales, así como también observamos la atribución de pericia por el simple hecho de ser joven. También, regresando a Pere, él mismo nos cuenta:

A mí me interesa aprender, no sé lo que me queda de vida, pero escucha, me estoy dando cuenta de que si no estás con estas cosas [refiriéndose a la tecnología y a las redes sociales], con eso práctico, te estás quedando hecho una ... «tifa». Te quedas atrás. Si no sabes de estas cosas, del teléfono y cosas de estas, ... escucha, yo a la juventud la veo allí, y están, clic, clic, clic, clic, y dejaría dar una solución. Ostras.... Eso es lo que yo quiero saber.

Esta cita resume muy bien el sentimiento que hemos observado. Hay una visión recurrente, tanto entre expertos como no expertos, de la tecnología (smartphones, tablets, redes sociales e informática en general) como algo propio de los jóvenes. Indirectamente, esto implica una visión «edadista» alrededor de las tecnologías y su uso, que debería ser superada si queremos potenciar la inclusión digital y evitar trasladar a la Red las desigualdades sociales por edad. Ya que como hemos visto los adultos mayores también participan del uso de la tecnología y emergen prácticas que son significativas para ellos. Relacionado con esto, transpira la idea de que es «negativo» no estar «en estas cosas». Si no estás con la tecnología te quedas detrás, ¿̇detrás de quién o de qué?, detrás de la gente más joven.

Sin embargo, lo que observamos en los participantes del estudio etnográfico es un tremendo deseo de vivir en el presente. Y el presente es innegablemente tecnológico. Para estar en ese presente tecnológico los participantes de esta investigación han construido sus propias prácticas -y aprendido con ello-, que son atractivas y significativas, y a partir de ellas se han forjado comunidades de aprendizaje fluidas, dinámicas, complejas, relacionales, afectivas y emocionales. En estas comunidades de aprendizaje no solo los actores sociales intervienen, también lo hacen los artefactos tecnológicos -en tanto que creaciones digitales de los propios participantes o en tanto que aparatos tecnológicos que incorporan en su día a día-. 
«HAGO DE TODO Y NO SÉ HACER FUNCIONAR NADA»: APRENDIZAJE AFECTIVO

Y RELACIONAL DE TECNOLOGÍAS DIGITALES EN ADULTOS MAYORES

ROSER BENEITO-MONTAGUT, ARANTZA BEGUERIA Y NIZAIÁ CASSIÁN

\section{Conclusiones}

Este artículo es un intento de entender y conocer cómo pensar sobre afecto, aprendizaje y comunidad y cómo estos elementos aparecen en las prácticas de aprendizaje en torno a las tecnologías digitales.

Una primera conclusión, tentativa, nos apunta a que los adultos mayores aprenden tecnologías digitales mediante relaciones afectivas -o, dicho de otro modo, el aprendizaje efectivo se produce a partir de relaciones que tienen un elemento relacional y emocional-. Las habilidades tecnológicas aparecen como una dimensión identitaria y los artefactos tecnológicos, y las habilidades que se demuestran con su manejo, se convierten en un aspecto que contribuye a la subjetividad del creador o del usuario.

En segundo lugar, poco a poco, creció en nosotras la sensación de que los lazos afectivos que se estaban describiendo eran importantes para el aprendizaje, para la continuidad del uso de la tecnología y para la pertenencia a la comunidad de práctica. Ya que la práctica en sí es lo que mantiene unido al grupo. En este sentido, aparecen dos tipos de lazos afectivos imbricados en las prácticas de aprendizaje: los que ya están ahí (como hijos/as y nietos/as) o los que surgen en el contexto de los centros socioculturales y se extienden por las redes sociales.

Finalmente, constatamos que diferentes formas de aprendizaje, y de ser, coexisten -dado que la práctica también implica una dimensión identitaria y/o subjetiva-. De manera que la idea de aprendizaje situado se expande para incorporar el aprendizaje que sucede en la Red y en interacciones sociales que ocurren también fuera del contexto de aprendizaje, que en este caso son los centros socioculturales de los barrios. Podemos entonces hablar de ecologías de aprendizaje (Barron, 2004). La metáfora ecológica aplicada al aprendizaje subraya la noción de la participación en la práctica y la pertenencia a la comunidad como una forma de «estar» y «ser», en la cual la naturaleza de la práctica es multidimensional (aprendizaje, relacional y afectiva), en vez de unidimensional, y emerge como parte de la interacción entre el significado de la práctica y el continuo trabajo identitario de los participantes en las determinadas situaciones.

\section{Bibliografía}

Aberton, H. (20I2) Material enactments of identities and learning in everyday community practices: implications for pedagogy. Pedagogy Cult. Soc., 20, II3-I36. https://doi.org/IO.IO 80/14681366.20I2.649418.

BARron, B. (2004) Learning Ecologies for Technological Fluency: Gender and Experience Differences. J. Educ. Comput. Res., 3I, I-36. https://doi.org/I0.2190/IN20-VVI2-4RB5-33VA.

Beneito-Montagut, R. (20II) Ethnography goes online: towards a user-centred methodology to research interpersonal communication on the internet. Qual. Res., II, 716-735.

Beneito-Montagut, R.; Begueria, A. y Cassián, N. (2017) Doing digital team ethnography: being there together and digital social data. Qual. Res., I7, 664-682.

Boulton-Lewis, G. M. (2010) Education and learning for the elderly: Why, how, what. Educ. Gerontol., 36, 213-228.

Diduca, D.; Partridge, C. y Heres, J. (2007) ict use and the elderly - cohort, lifestage or just irrelevant? En Information and Communications Technologies in Society: E-Living in a Digital Europe (p. 88). 
«HAGO DE TODO Y NO SÉ HACER FUNCIONAR NADA»: APRENDIZAJE AFECTIVO Y RELACIONAL DE TECNOLOGÍAS DIGITALES EN ADULTOS MAYORES ROSER BENEITO-MONTAGUT, ARANTZA BEGUERIA Y NIZAIÁ CASSIÁN

Erickson, F. (1985) Qualitative Methods in Research on Teaching. Occasional Paper n. ${ }^{\circ}$ 8I.

Fenwick, T. y LANDRI, P. (20I2) Materialities, textures and pedagogies: socio-material assemblages in education. Pedagogy Cult. Soc., 20, I-7.

Fernández-Ardèvol, M. y Prieto, L. A. (20I2) Mobile telephony and older people: Exploring use and rejection. Interact. Stud. Commun. Cult., 3, 9-24.

Goffman, E. (2006) Stigma: Notes on the management of spoiled identity. Simon and Schuster.

Hughes, J.; Jewson, N. y Unwin, L. (2013) Communities of Practice: Critical Perspectives. Routledge.

Jaeger, P. T. y Xie, B. (2009) Developing online community accessibility guidelines for persons with disabilities and older adults. J. Disabil. Policy Stud., 20, 55-63.

Lave, J. y Wenger, E. (2002) Legitimate peripheral participation in communities of practice. Support. Lifelong Learn., I, III-I26.

Lave, J. y Wenger, E. (1991) Situated learning: Legitimate peripheral participation. Cambridge University Press.

LeIst, A. K. (2013) Social media use of older adults: a mini-review. Gerontology, 59, 378-384.

MADDEN, M. (20IO) Older adults and social media.

Mathisen, A. y Nerland, M. (20I2) The pedagogy of complex work support systems: infrastructuring practices and the production of critical awareness in risk auditing. Pedagogy Cult. Soc., 20, 7I-9I. https://doi.org/I0.1080/I4681366.20I2.649416.

Miles, M. B. y Huberman, A. M. (1994) Qualitative data analysis: An expanded sourcebook. sage.

Policies for Ageing Well with ict [www Document], n.d. Digit. Single Mark. url https:// ec.europa.eu/digital-single-market/en/policies/ageing-well-ict (accessed I2.I0.I7).

SEgal, L. (2008) After Judith Butler: identities, who needs them? Subjectivity, 25, 38I-394.

SELWYN, N. (2004) The information aged: A qualitative study of older adults' use of information and communications technology. J. Aging Stud., 18, 369-384. https://doi.org/Io.1016/j. jaging.2004.06.008.

Selwyn, N.; Gorard, S.; Furlong, J. y Madden, L. (2003) Older adults' use of information and communications technology in everyday life. Ageing Amp Soc., 23, 56I-582. https://doi. org/Io.roi7/Sor44686Хo3001302.

Sørensen, E. (2009) The materiality of learning: Technology and knowledge in educational practice. Cambridge University Press.

Timmers, P. (2008) EU e-inclusion policy in context. info IO, I2-I9. https://doi.org/Io.IIo8/ I4636690810904670.

Tsai, H. S.; Shillair, R.; Cotten, S. R.; Winstead, V. y Yost, E. (20I5) Getting grandma online: are tablets the answer for increasing digital inclusion for older adults in the us? Educ. Gerontol., 4I, 695-709.

Wenger, E. (1998a) Communities of practice: Learning, meaning, and identity. Cambridge University Press.

Wenger, E. (1998b) Communities of practice: Learning as a social system. Syst. Think., 9, 2-3. Wetherell, M. (2012) Affect and emotion: A new social science understanding. Sage Publications. 